\begin{tabular}{|c|c|}
\hline Title & Subblock processing in MMSE-FDE under fast fading environments \\
\hline Author(s) & Kambara, Keiichi; Nishimoto, Hiroshi; Nishimura, Toshihiko; Ohgane, Takeo; Ogawa, Y asutaka \\
\hline Citation & $\begin{array}{l}\text { IEEE Journal on Selected A reas in Communications, 26(2), 359-365 } \\
\text { https://doi.org/10.1109/JSA C.2008.080212 }\end{array}$ \\
\hline Issue Date & $2008-02$ \\
\hline Doc URL & http:/hdl. handle.net/2115/33044 \\
\hline Rights & $\begin{array}{l}\text { () } 2008 \text { IEEE. Personal use of this material is permitted. However, permission to reprint/republish this material for } \\
\text { advertising or promotional purposes or for creating new collective works for resale or redistribution to servers or lists, } \\
\text { or to reuse any copyrighted component of this work in other works must be obtained from the IEEE. }\end{array}$ \\
\hline Type & article \\
\hline File Information & 04444766.pdf \\
\hline
\end{tabular}

Instructions for use 


\title{
Subblock Processing in MMSE-FDE Under Fast Fading Environments
}

\author{
Keiichi Kambara, Hiroshi Nishimoto, Student Member, IEEE, Toshihiko Nishimura, Member, IEEE, \\ Takeo Ohgane, Member, IEEE, and Yasutaka Ogawa, Senior Member, IEEE
}

\begin{abstract}
Frequency domain equalization (FDE) has been studied for reducing inter-symbol interference (ISI) caused by frequency selective fading in single carrier systems. When a high-mobility terminal exists in the system, the channel state may change within a DFT block. Then, the ISI reduction performance of FDE degrades since cyclicity of the channel matrix is lost. We propose to divide a received data block into multiple subblocks to decrease the channel transition within the DFT block in fast fading environments. Also, to satisfy periodicity of the received signal in each subblock, we introduce a pseudo cyclic prefix technique. The results of numerical analysis show that the proposed method can effectively decrease the error floor in fast fading environments.
\end{abstract}

Index Terms-Fast fading, single carrier transmission, MMSEFDE, unique word, subblock processing

\section{INTRODUCTION}

$\mathbf{N}$ OW, high data-rate services over $100 \mathrm{Mbps}$ are discussed in standardization processes for WiFi, WiMAX, and beyond third generation. These broadband systems suffer from frequency selective fading, for which a multi-carrier transmission such as orthogonal frequency division multiplexing $(\mathrm{OFDM})$ is very effective [1]. However, OFDM systems have the problem of a high peak-to-average-power-ratio (PAPR) [2].

Although a single carrier transmission has the advantage of a low PAPR, inter-symbol interference (ISI) caused by frequency selective fading must be reduced. Frequency domain equalization (FDE) is a simple technique to reduce ISI for severe frequency selectivity [3]. FDE is based on the cyclic signal property within the target block. This means that the channel must remain constant in the period. Thus, in fast fading environments, channel transition in an FDE block degrades the equalization performance.

A reasonable solution for the issue is a method controlling transmission block size adaptively. By changing the transmission block size according to the Doppler frequency, the channel transition within the block can be reduced. However, this method requires Doppler frequency information at the transmitter, i.e., closed-loop control. In addition, the shortened transmission block size with a constant cyclic prefix (CP) size leads to lower transmission efficiency.

In this paper, we propose subblock FDE processing at the receiver side without reducing transmission block size. In

Manuscript received April 3, 2007; revised August 30, 2007. This work was supported in part by a Research Fellowship for Young Scientists from the Japan Society for the Promotion of Science. This paper was partially presented at IEEE VTC 2007 Spring held in Dublin, April 23-25, 2007.

The authors are with the Graduate School of Information Science and Technology, Hokkaido University, Sapporo, 060-0814 Japan (e-mail: kan@micl.ist.hokudai.ac.jp; \{hn,nishim,ohgane,ogawa\}@ist.hokudai.ac.jp).

Digital Object Identifier 10.1109/JSAC.2008.080212. this method, inter-subblock interference (ISBI) occurs in each subblock. Therefore, we apply a CP reconstruction scheme (hereinafter referred to as "pseudo CP generation") to satisfy periodicity in a subblock DFT window [4].

The rest of the paper is organized as follows. In Section II, the fundamental formulation of FDE and conventional countermeasures for fast fading environments are reviewed. Then, the proposed method is described in Section III. After numerical analysis in Section IV, Section V concludes the paper.

\section{FDE UNDER FAST FADING ENVIRONMENTS}

\section{A. FDE and Cyclicity of Channel Matrix}

Let us consider a single carrier system with FDE per $N$ symbol block at the receiver side. To maintain periodicity of the received signal within a DFT window, we add a CP longer than the maximum symbol delay. Here, assuming a multipath channel with $L$ symbol-spaced paths, the CP length $N_{P}$ is set as $N_{P} \geq L$. When we define an $N$-dimensional transmit signal vector $s=\left[s_{0}, \ldots, s_{N-1}\right]^{T}$ and an $N$-dimensional noise vector, the $N$-dimensional received signal vector after $\mathrm{CP}$ removal is given by [5]

$$
r=H s+n,
$$

where $\boldsymbol{H}$ is the $N \times N$ channel matrix expressed by

$$
\begin{aligned}
& \boldsymbol{H}= \\
& {\left[\begin{array}{ccccccc}
h_{0,0} & 0 & \cdots & 0 & \cdots & h_{2,-2} & h_{1,-1} \\
h_{1,0} & h_{0,1} & \cdots & 0 & \cdots & h_{3,-2} & h_{2,-1} \\
\vdots & \vdots & \ddots & \vdots & \ddots & \vdots & \vdots \\
h_{L-1,0} & h_{L-2,1} & \cdots & h_{0, N-L} & \cdots & 0 & 0 \\
\vdots & \vdots & \ddots & \vdots & \ddots & \vdots & \vdots \\
0 & 0 & \cdots & h_{L-2, N-L} & \cdots & h_{0, N-2} & 0 \\
0 & 0 & \cdots & h_{L-1, N-L} & \cdots & h_{1, N-2} & h_{0, N-1}
\end{array}\right]}
\end{aligned}
$$

The first index $i$ and the second index $j$ of $h_{i, j}$ correspond to multipath number and transmitting time, respectively. Note that the definition of the time index is different from that in [5] where receiving time index is used, since it is useful to explain the equivalent noise power in Appendix A.

Transforming (1) into the frequency domain using the DFT matrix $\boldsymbol{F}$ yields

$$
\begin{aligned}
\boldsymbol{F r} & =\boldsymbol{F} \boldsymbol{H} \boldsymbol{s}+\boldsymbol{F} \boldsymbol{n} \\
& =\boldsymbol{F} \boldsymbol{H} \boldsymbol{F}^{H} \boldsymbol{F} \boldsymbol{s}+\boldsymbol{F n},
\end{aligned}
$$


where $\boldsymbol{F}^{H} \boldsymbol{F}=\boldsymbol{I}_{N}$ because $\boldsymbol{F}$ is a unitary matrix. When the channel is time-invariant within the block, i.e., $h_{l, 0}=$ $\cdots=h_{l, N-1}=h_{l}$ for $l=0, \ldots, L-1, \boldsymbol{H}$ becomes a cyclic matrix. Thus, $\boldsymbol{H}$ can be diagonalized by $\boldsymbol{F}$ as $\boldsymbol{F} \boldsymbol{H} \boldsymbol{F}^{H}=$ $\boldsymbol{D}=\operatorname{diag}\left(d_{0}, \ldots, d_{N-1}\right)$, where we have

$$
\left[\begin{array}{c}
d_{0} \\
\vdots \\
d_{N-1}
\end{array}\right]=\boldsymbol{F}\left[\begin{array}{c}
h_{0} \\
\vdots \\
h_{N-1}
\end{array}\right] \text {. }
$$

Then, (4) can be rewritten as

$$
\begin{aligned}
\boldsymbol{F} \boldsymbol{r} & =\boldsymbol{D} \boldsymbol{F} \boldsymbol{s}+\boldsymbol{F} \boldsymbol{n} \\
\tilde{\boldsymbol{r}} & =\boldsymbol{D} \tilde{\boldsymbol{s}}+\tilde{\boldsymbol{n}} \\
\tilde{r}_{k} & =d_{k} \tilde{s}_{k}+\tilde{n}_{k},
\end{aligned}
$$

where $\tilde{\boldsymbol{r}}, \tilde{\boldsymbol{s}}$, and $\tilde{\boldsymbol{n}}$ are the received signal, the transmitted signal, and the noise vectors represented in the frequency domain, respectively, and $\tilde{r}_{k}, \tilde{s}_{k}$, and $\tilde{n}_{k}$ correspond to each component as well. Equation (8) indicates that the signal components in the frequency domain are mutually independent. Thus, minimum mean square error (MMSE) estimation of the transmitted signal can be independently performed at each frequency. The MMSE weight at the $k$ th frequency point is expressed by $w_{k}=d_{k}^{*} /\left(\left|d_{k}\right|^{2}+\sigma^{2}\right)$, where $\sigma^{2}=E\left[\tilde{n}_{k} \tilde{n}_{k}^{*}\right]$ denotes the noise power, and equalization can be easily accomplished by $\hat{\tilde{s}}_{k}=w_{k} \tilde{r}_{k}$. Finally, by applying IDFT to the MMSE-FDE output, we can estimate the transmitted data sequence.

\section{B. Residual ISI Component Due to Fast Fading}

In fast fading environments, cyclicity of the channel matrix $\boldsymbol{H}$ is no longer valid due to channel transition within the FDE block. Thus, $\boldsymbol{F} \boldsymbol{H} \boldsymbol{F}^{H}$ includes non-diagonal components as

$$
\boldsymbol{F} \boldsymbol{H} \boldsymbol{F}^{H}=\boldsymbol{D}^{\prime}+\boldsymbol{E},
$$

where $\boldsymbol{D}^{\prime}$ expresses the diagonal component and $\boldsymbol{E}$ is an $N \times$ $N$ matrix having off-diagonal elements only. Substituting (9) into (4) yields

$$
\begin{aligned}
\tilde{\boldsymbol{r}} & =\boldsymbol{D}^{\prime} \tilde{\boldsymbol{s}}+\tilde{\boldsymbol{n}}+\boldsymbol{E} \tilde{\boldsymbol{s}} \\
\tilde{r}_{k} & =d_{k}^{\prime} \tilde{s}_{k}+\tilde{n}_{k}+\sum_{j=0}^{N-1} e_{(k, j)} \tilde{s}_{j},
\end{aligned}
$$

where $e_{(k, j)}$ is the $(k, j)$ th element of $\boldsymbol{E}$. The third terms on the right-hand sides of these equations correspond to interfrequency interference components, or may be regarded as residual ISI components in the time domain. Thus, the MMSE estimation is only achieved by solving an inverse problem of the $N \times N$ matrix $\left(\boldsymbol{D}^{\prime}+\boldsymbol{E}\right)$ so that the numerical complexity grows enormously as the block size increases.

However, if the third term in (11) is relatively small compared to the first term, we can still apply the conventional FDE by regarding the third term as additional noise to $\tilde{n}_{k}$

$$
\tilde{r}_{k}=d_{k}^{\prime} \tilde{s}_{k}+\tilde{n}_{k}^{\prime} \text {. }
$$

In this case, the MMSE weight would be obtained using equivalent noise power

$$
\sigma_{e, k}^{2}=\sigma^{2}+\left|\sum_{j=0}^{N-1} e_{(k, j)} \tilde{s}_{j}\right|^{2} .
$$

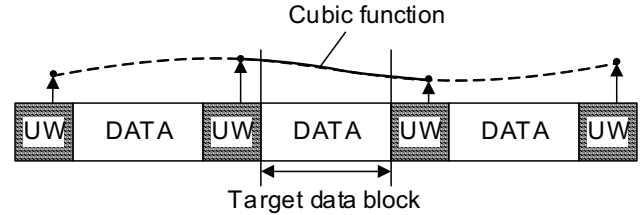

(a) Third order interpolation

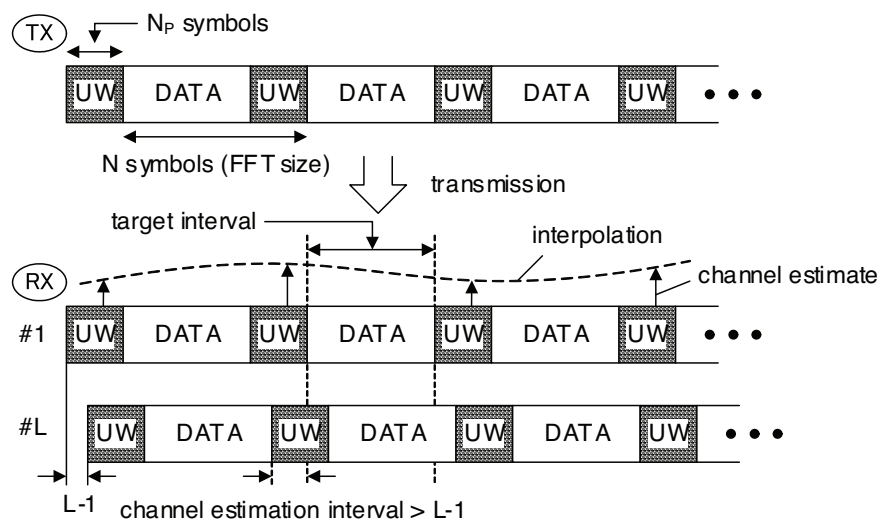

(b) Transmitted signal block and channel estimation

Fig. 1. Channel estimation.

Since the transmitted signal is unknown at this moment, $\left|\sum e_{(k, j)} \tilde{s}_{j}\right|^{2}$ cannot be obtained. Therefore, we consider the ensemble average $E\left[\left|\sum e_{(k, j)} \tilde{s}_{j}\right|^{2}\right]$ instead. Assuming that $\tilde{s}_{j}$ is uncorrelated over frequencies and that its average power at each frequency is equal to $P_{s}$, we have

$$
E\left[\tilde{s}_{i} \tilde{s}_{j}^{*}\right]=\left\{\begin{array}{ll}
P_{s} & (i=j) \\
0 & (i \neq j)
\end{array} .\right.
$$

Then, the equivalent noise power at the $k$ th frequency is given by

$$
\sigma_{e, k}^{2}=\sigma^{2}+P_{s} \sum_{j=0}^{N-1}\left|e_{(k, j)}\right|^{2} .
$$

Thus, FDE can be applied with less performance degradation in a high SNR region as shown later.

Equation (15) requires all elements in $\boldsymbol{E}$ so calculating $\boldsymbol{F} \boldsymbol{H} \boldsymbol{F}^{H}$ ( $N^{3}$-order complexity) is also necessary. To ease the calculation load, we use the following approximation. Defining expansion forms of the DFT matrix $\boldsymbol{F}$ and non-diagonal matrix $\boldsymbol{E}$ as $\boldsymbol{F}=\left[\boldsymbol{f}_{0}, \ldots, \boldsymbol{f}_{N-1}\right]^{T}$ and $\boldsymbol{E}=\left[\boldsymbol{e}_{0}, \ldots, \boldsymbol{e}_{N-1}\right]^{T}$, the equivalent noise power is approximated as

$$
\begin{aligned}
\sigma_{e, k}^{2} & =\sigma^{2}+P_{s} \boldsymbol{e}_{k}^{H} \boldsymbol{e}_{k} \\
& \simeq \sigma^{2}+P_{s} \beta\left|\boldsymbol{f}_{k}^{T}\left(\boldsymbol{h}_{N-1}-\boldsymbol{h}_{0}\right)\right|^{2},
\end{aligned}
$$

where $\beta$ is a constant, and $\boldsymbol{h}_{0}=\left[h_{0,0}, \ldots, h_{L-1,0}, 0, \ldots, 0\right]^{T}$ and $\boldsymbol{h}_{N-1}=\left[h_{0, N-1}, \ldots, h_{L-1, N-1}, 0, \ldots, 0\right]^{T}$ are impulse response vectors at the zeroth and $(N-1)$ th symbol timings. The derivation is described in Appendix A.

\section{Channel Estimation Using Known Pilot SEQUENCE}

Since we consider a fast fading environment, channel state information (CSI) in each FFT block must be estimated. The conventional CP (i.e., a copy of the tail in the transmitted block) is basically unknown before data are detected using the 


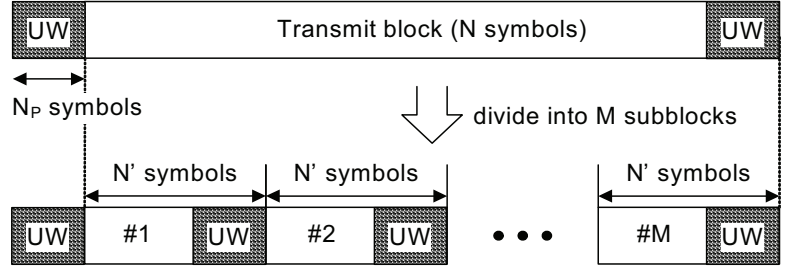

Fig. 2. Block division transmission.

FDE. Therefore, inserting a unique word (UW) as a known pilot sequence instead of the CP has been proposed [6]- [8]. In this case, the data block size is decreased to $N-N_{P}$ for satisfying periodicity within the DFT window as shown in Fig. 1. When $N_{P} \geq 2 L-1$, we have a part of more than $(L-1)$ symbols, which is uninterfered from the data block and includes the UW only, in the received signal. Thus, the CSI can be simply estimated exploiting this period.

The MMSE algorithm for channel estimation in the time domain is applied to this $\left(N_{P}-L\right)$-symbol sequence. Using the $\left(N_{P}-L\right)$-dimensional received signal vector $\boldsymbol{z}$, the $L$ dimensional channel vector $\boldsymbol{h}_{\mathrm{uw}}$, and the $\left(N_{P}-L\right) \times L$ transmit signal matrix in this interval $Q$ where the $i$-th column vector is an $i$-symbol-cyclic-shifted partial $\mathrm{UW}$ sequence, the square error function $J$ is defined as

$$
J=\left(\boldsymbol{z}-\boldsymbol{Q} \boldsymbol{h}_{\mathrm{uw}}\right)^{H}\left(\boldsymbol{z}-\boldsymbol{Q} \boldsymbol{h}_{\mathrm{uw}}\right),
$$

where $\boldsymbol{h}_{\mathrm{uw}}$ is assumed as the time-invariant channel response within the target signal sequence. Then, the $\boldsymbol{h}_{\mathrm{uw}}$ can be estimated by minimizing $J$ [9] as

$$
\boldsymbol{h}_{\mathrm{uw}}=\left(\boldsymbol{Q}^{H} \boldsymbol{Q}\right)^{-1} \boldsymbol{Q}^{H} \boldsymbol{z} .
$$

We need to know the channel response at the central position of the block/subblock for FDE. In addition, the approximation of the equivalent noise power estimation requires the channel responses at the head and tail as in (35) and (42). Moreover, all responses within the pseudo $\mathrm{CP}$ part are also needed. Therefore, the channel estimates at several points are necessary in total. Since the UW for channel estimation is only located at pre- and post-data blocks, we obtain channel estimates within the data block by interpolation. In this paper, third order interpolation is used as shown in Fig. 1(b). By using channel estimates at four UWs (two in the past and two in the future of the target data block), the channel within the data block is interpolated with a cubic function. (See subsection 3-1 in [10] for the detail.) Then, the channel responses at the other required points are obtained from the curve. When applying the FDE, we used the central channel response $\boldsymbol{h}_{\text {cent }}=\left[h_{0, \text { cent }}, \ldots, h_{L-1, \text { cent }}, 0, \ldots, 0\right]^{T}$ in the target block to calculate $d_{0}, \ldots, d_{N-1}$, i.e., $\left[d_{0}, \ldots, d_{N-1}\right]^{T} \simeq \boldsymbol{F} \boldsymbol{h}_{\text {cent }}$.

\section{Block Division Technique}

\section{A. Block Division Transmission}

The simplest way to reduce the channel transition observed within the FDE block is to shorten the block size. If the maximum Doppler frequency (or a related value) is known at the transmitter, we can divide the block into $M$ subblocks in advance as illustrated in Fig. 2 where $M$ is adaptively determined so as to suppress the equivalent noise power to

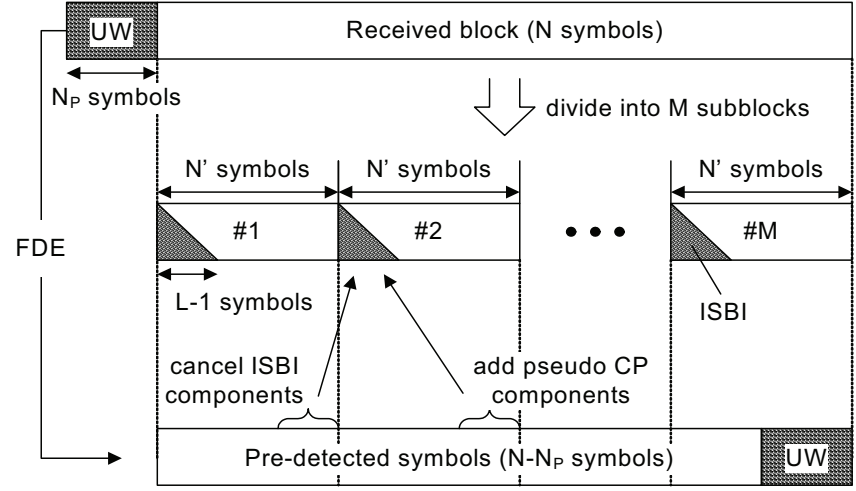

Fig. 3. Concept of subblock processing at receiver side.

a required level. A UW insertion to each subblock also improves tracking capability of the channel transition. Thus, the performance degradation due to fast fading can be reasonably suppressed.

In this technique, however, the transmitter must know the fading speed information so that a closed-loop control based on feedback from the receiver is required. In addition, when the maximum Doppler frequency is very high, the number of subblocks becomes very large. More frequent insertion of the UW makes the transmission efficiency lower. Our objective is to avoid these problems. The proposed method is described next.

\section{B. Subblock Processing at Receiver Side}

In this paper, we propose subblock processing, which shortens the FDE block size equivalently without changing the transmitted block format. The concept is shown in Fig. 3. The basic idea of the proposed method is to divide the whole received block into $M$ subblocks at the receiver side. Then, as shown in the middle part of Fig. 3, the top $L-1$ symbols of each subblock contain ISBI components. Therefore, reducing the ISBI is required first. In addition, the received signal in the subblock needs to have periodicity so that the tail of subblock data convoluted with the multipath channel should be added to the top of the subblock. In the paper, the following pseudo $\mathrm{CP}$ generation [4] is used for these requirements.

At first, the MMSE-FDE is applied to the whole received block to obtain a tentative decision $\hat{s}=\left[\hat{s}_{0}, \ldots, \hat{s}_{N-1}\right]^{T}$. The number of symbols within a subblock is $N^{\prime}=N / M$. Then, the received signal at the $i$ th $(0 \leq i \leq L-2)$ symbol timing of the $m$ th subblock is written as

$$
\begin{aligned}
r_{m, i}= & \sum_{l=0}^{i} h_{l, i+N^{\prime} m} s_{m, i-l} \\
& +\sum_{l=i+1}^{L-1} h_{l, i+N^{\prime} m} s_{m-1, i+N^{\prime}-l}+n_{i+N^{\prime} m},
\end{aligned}
$$

where $s_{m, j}$ is the $j$ th transmitted symbol of the $m$ th subblock. Note that the second term in (20) corresponds to ISBI components. This part can be cancelled using the tentative decision $\hat{s}$ and the estimated channel response $\hat{h}_{l, t}$ as

$$
r_{m, i}^{\prime}=r_{m, i}-\sum_{l=i+1}^{L-1} \hat{h}_{l, i+N^{\prime} m} \hat{s}_{m-1, i+N^{\prime}-l},
$$


TABLE I

SIMULATION PARAMETERS

\begin{tabular}{c|c}
\hline \hline Modulation & QPSK for data (BPSK for UW) \\
\hline Block size $N$ & $\begin{array}{c}256,512,1024,2048,4096,8192 \text { symbols } \\
\text { (including 48-symbol UW) }\end{array}$ \\
\hline Number of paths $L$ & 16 (symbol-spaced, equal-level) \\
\hline Fading model & $\begin{array}{c}\text { Rayleigh (Jakes' model) } \\
\text { Uncorrelated between paths }\end{array}$ \\
\hline Number of subblocks $M$ & $2,4,8$ \\
\hline FEC & Binary CC (4 states, $\left.R_{c}=1 / 2\right)$ \\
\hline Decoder & Soft Viterbi algorithm \\
\hline Number of trial blocks & 100000 \\
\hline \hline
\end{tabular}

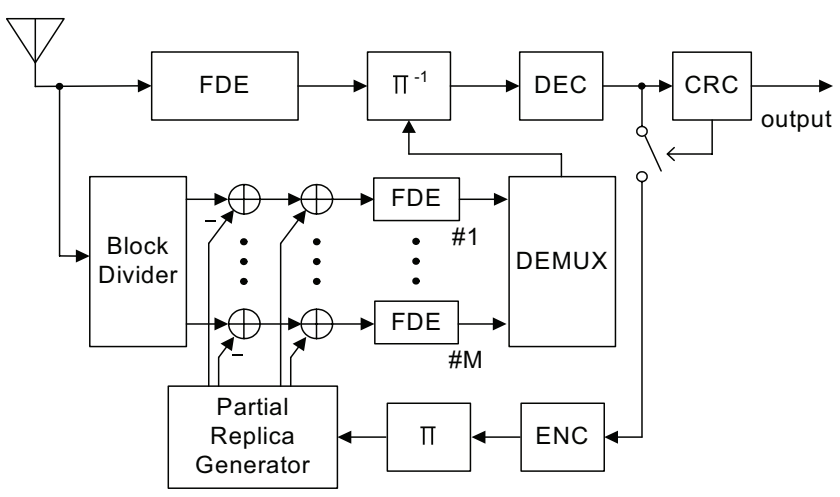

Fig. 4. Receiver block diagram.

where $\hat{s}_{m-1, j}$ denotes the tentative decision of the $j$ th symbol of the $(m-1)$ th subblock. Next, adding the tail part of the tentative decision of the $m$ th subblock convoluted with the channel responses to $r_{m, i}^{\prime}$ yields

$$
r_{m, i}^{\prime \prime}=r_{m, i}^{\prime}+\sum_{l=i+1}^{L-1} \hat{h}_{l, i+N^{\prime} m} \hat{s}_{m, i+N^{\prime}-l} .
$$

Consequently, the pseudo received signal satisfies periodicity within each subblock when the tentative decision and the estimated channel are reasonably correct. After the above pseudo CP procedure, subblock-based FDE is done in each subblock. Then, each output is demultiplexed and passed to a decoder. Although this technique is affected by the accuracy of the pseudo CP (i.e., channel estimates and tentative decisions), similar improvement to block division transmission can be expected.

\section{NUMERICAL ANALYSiS}

\section{A. Simulation Environment}

The performance of the proposed system was numerically evaluated using computer simulations. The simulation parameters are shown in Table I. In the following discussions, we use the normalized Doppler frequency $F_{D}$, which is a product of the maximum Doppler frequency $f_{D}$ and the block length $N T_{s}$ ( $T_{s}$ : the symbol duration), as a fading speed measure.

The receiver configuration is shown in Fig. 4. In this paper, we consider data transmission based on automatic repeat request (ARQ). Thus, use of a cyclic redundancy check (CRC) code is assumed to enable a block error check for ARQ. First, the whole received block is equalized and decoded. If no errors

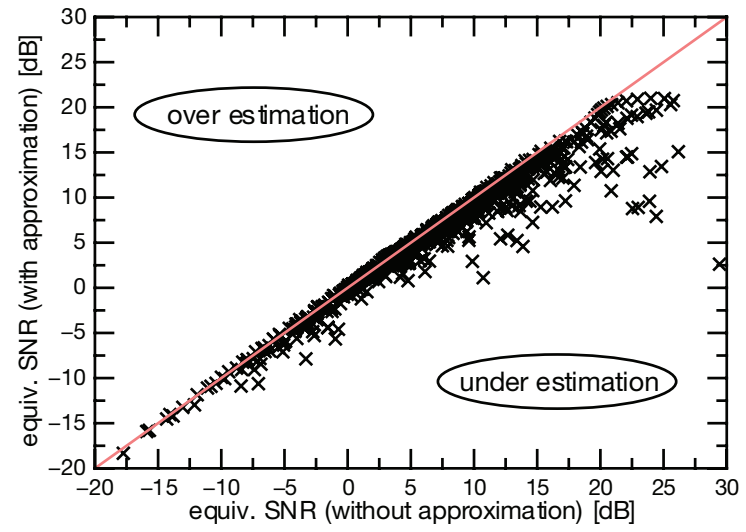

(a) The distribution of the equivalent SNR

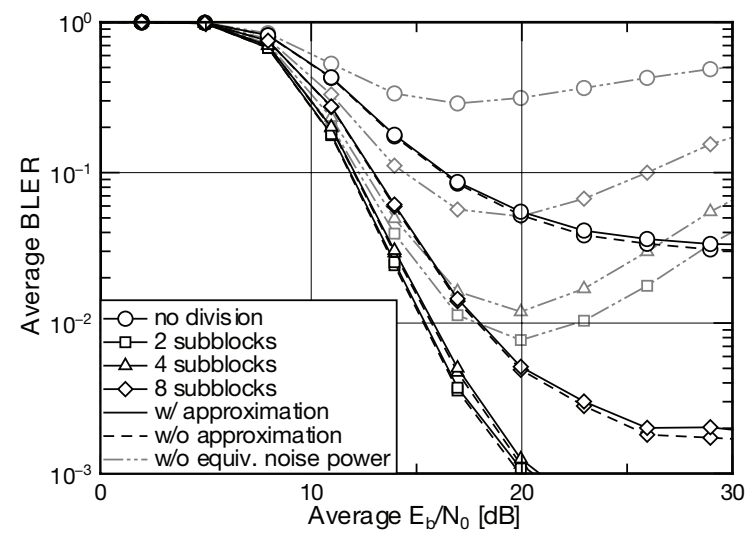

(b) BLER performance

Fig. 5. Comparison of cases with and without approximation for calculating equivalent noise power when the block size is 256 symbols and $F_{D}=0.3$ (estimated CSI).

are found in a cyclic redundancy check, the decoded data are output. Otherwise, the block is divided into subblocks, and then the pseudo $\mathrm{CP}$ processing and the subblock FDE are applied. Finally, each output is demultiplexed and decoded.

\section{B. Validity of Approximation on Equivalent Noise Power}

First, we evaluate the validity of the approximation when we calculate the equivalent noise power as in (17). Figure 5(a) shows a correlation chart of the equivalent SNR with and without the approximation. It can be seen that the approximation works well in the low SNR region, i.e., high impact region of equivalent noise. Figure 5(b) shows the block error rate (BLER) performance with and without the approximation where we recalculate the equivalent noise power in the subblock FDE. To demonstrate the effectiveness of using the equivalent noise power, the performance with the noise power only (ignoring the third term in (11)) is also shown. If we do not use the equivalent noise power, error floors can be seen, and they become worse in the higher SNR region due to underestimating the noise power. In contrast, the BLER performance improves considerably by incorporating the equivalent noise power. In addition, very little degradation due to the approximation is observed. Therefore, in the following, we apply the approximation instead of the strict calculation for both conventional and subblock FDE. 


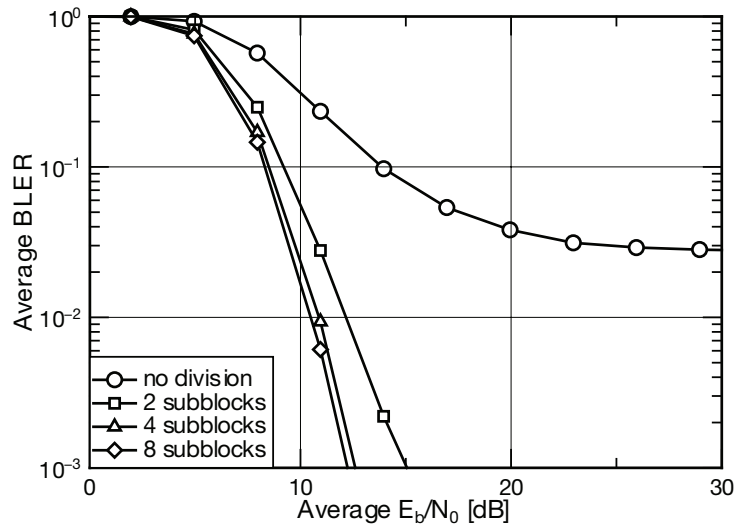

(a) CSI and tentative decisions are perfect when $F_{D}=0.3$.

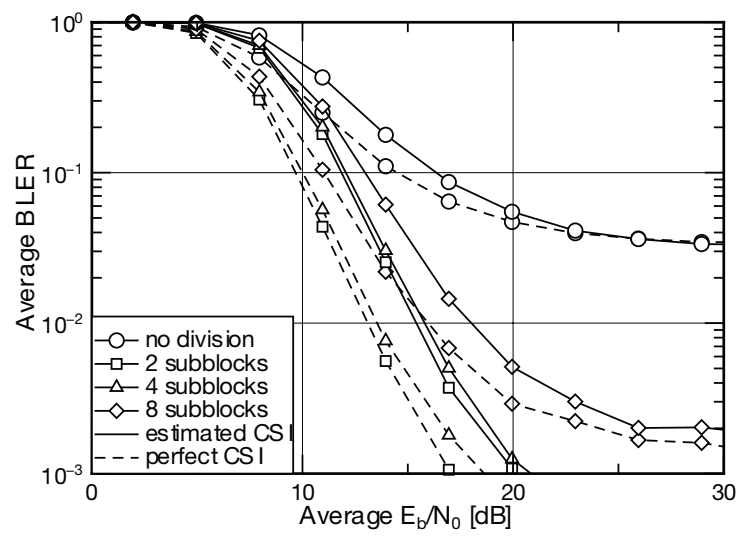

(b) $F_{D}=0.3$

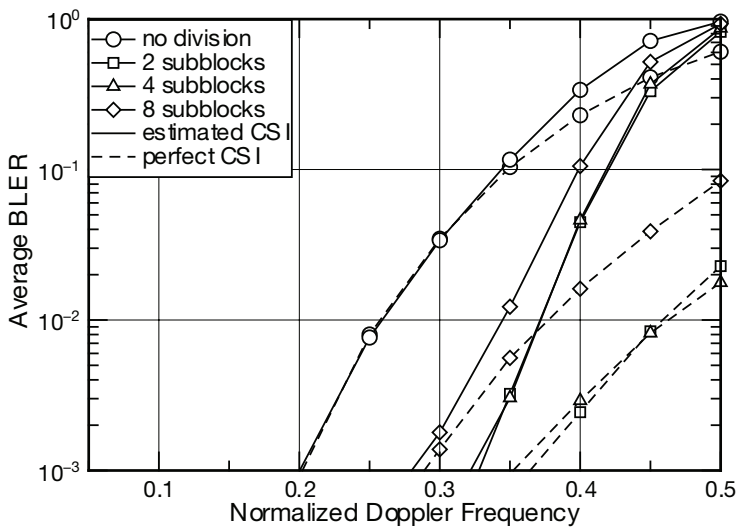

(c) Average $E_{b} / N_{0}=30 \mathrm{~dB}$

Fig. 6. BLER performance when the block size is 256 symbols.

\section{BLER Performance of Proposed Method}

The BLER performance is shown in Fig. 6 when the block size is 256 symbols. Figure 6(a) shows the case of perfect pseudo $\mathrm{CP}$ when $F_{D}=0.3$. From the figure, a monotonic improvement of BLER with decreasing subblock size can be seen. However, the imperfect pseudo $\mathrm{CP}$ in a realistic situation degrades the performance, and a trade-off between subblock size and performance is observed. To be specific, the best performance is obtained by two-subblock processing.

Basically, FDE with a smaller subblock size becomes more tolerant to $F_{D}$. However, the performance of the proposed method is affected by the accuracy of the pseudo CP, which depends on both the channel estimates and the tentative decisions as mentioned above. Comparing Fig. 6(a) with Fig. 6(b)

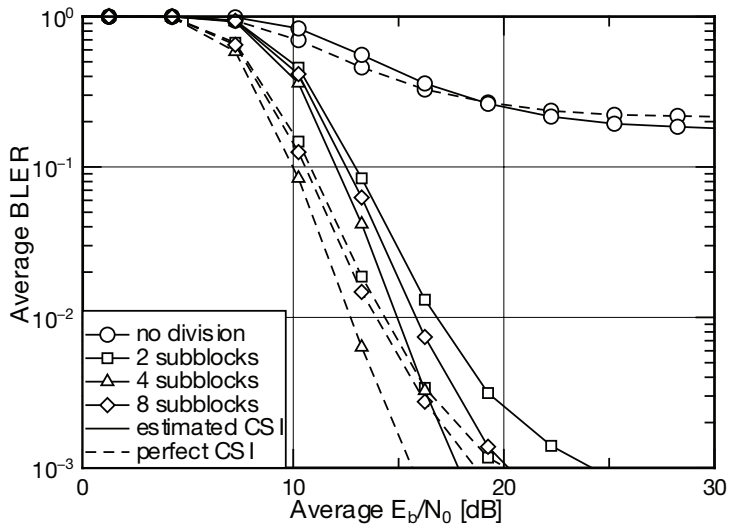

(a) $F_{D}=0.3$

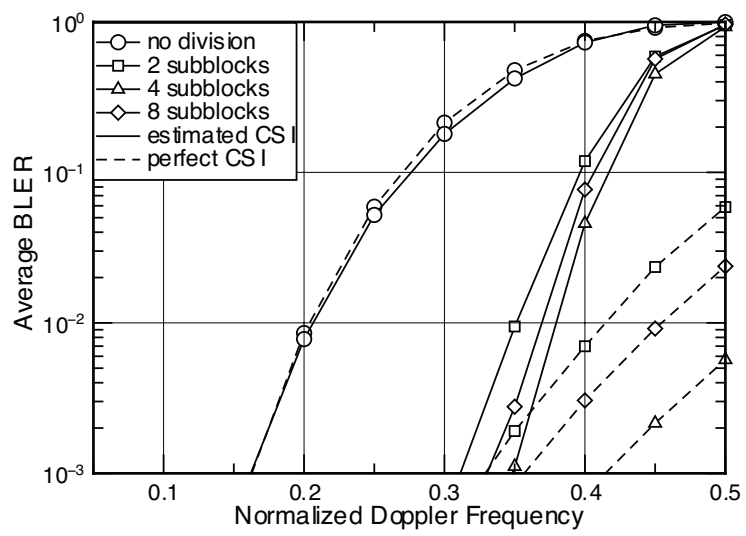

(b) Average $E_{b} / N_{0}=30 \mathrm{~dB}$

Fig. 7. BLER performance when the block size is 1024 symbols.

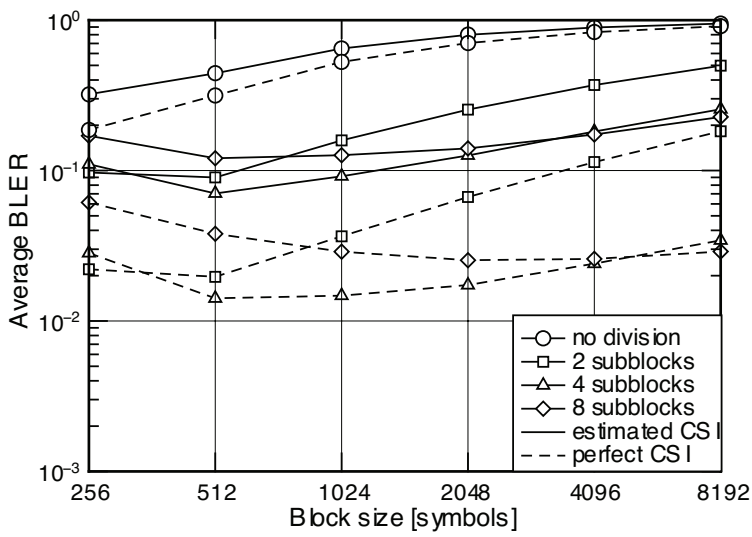

Fig. 8. Block size vs. BLER when average $E_{b} / N_{0}=12 \mathrm{~dB}$ and $F_{D}=0.3$.

gives us a conclusion that reconstructed pseudo $\mathrm{CP}$ is not so accurate due to errors in channel estimation and tentative decisions. Such an inaccurate pseudo CP adds errors in the received signal and destroys the cyclicity/periodicity of the subblock. Thus, it can be said that the ratio of the pseudo $\mathrm{CP}$ to the subblock size highly affects the capability of subblock FDE. In other words, the proposed scheme has the trade-off between the number of subblocks and error propagation due to the inaccuracy of pseudo CP. Consequently, two-subblock processing provides the best performance for $F_{D}=0.3$.

Figure 6(c) shows the BLER performance versus $F_{D}$ when $E_{b} / N_{0}=30 \mathrm{~dB}$. When assuming a required BLER of $10^{-2}$, two-subblock processing in the estimated CSI case provides 
the best performance for $F_{D} \geq 0.3$ and is applicable until $F_{D}=0.37$, which corresponds to about 1.5 times the speed applicable in the conventional FDE case, i.e., $F_{D}=0.25$.

Next, we show the performance of different block size with the same $F_{D}$. When $F_{D}$ is the same in the different block sizes, the channel estimation accuracy is almost the same. So, we can discuss the relationship between the optimum subblock number and pseudo CP ratio. Figure 7 shows the BLER performance when the block size is 1024 symbols. In this case, the best BLER performance is given by four-subblock processing. Since each subblock size is larger than that of the previous case, the ratio of the pseudo $\mathrm{CP}$ in the subblock becomes smaller due to the same CP size. Thus, the effect of errors included in the pseudo CP is also lower. Consequently, the number of subblocks giving the best performance is shifted to four with an increased block size.

Finally, the BLER performance versus block size is shown in Fig. 8 when $E_{b} / N_{0}=12 \mathrm{~dB}$ and $F_{D}=0.3$. The performance of the larger block size tends to be better as the number of subblocks increases. This property can be said to support the above discussion on the effect of the pseudo $\mathrm{CP}$ accuracy. Although the optimum number of subblocks depends on the block size, four-subblock processing shows robust performance in the given $F_{D}$.

\section{CONCLUSION}

In this paper, we have proposed subblock processing to reduce the effect of the channel transition within the FDE block. The numerical analysis has shown that the proposed method can effectively decrease the error floor in fast fading environments, and this method provides a 1.5 -fold increase in a tolerable Doppler frequency for BLER of $10^{-2}$ when the transmitted block size is 256 symbols. The optimal number of subblocks has been shown to change in accordance with the block size.

\section{APPENDIX}

\section{A. Approximation of equivalent noise power}

Let us derive the approximation of the equivalent noise power. As in (2), the channel matrix $\boldsymbol{H}$ is expressed as

$$
\begin{aligned}
& \boldsymbol{H}= \\
& {\left[\begin{array}{ccccccc}
h_{0,0} & 0 & \cdots & 0 & \cdots & h_{2,-2} & h_{1,-1} \\
h_{1,0} & h_{0,1} & \cdots & 0 & \cdots & h_{3,-2} & h_{2,-1} \\
\vdots & \vdots & \ddots & \vdots & \ddots & \vdots & \vdots \\
h_{L-1,0} & h_{L-2,1} & \cdots & h_{0, N-L} & \cdots & 0 & 0 \\
\vdots & \vdots & \ddots & \vdots & \ddots & \vdots & \vdots \\
0 & 0 & \cdots & h_{L-2, N-L} & \cdots & h_{0, N-2} & 0 \\
0 & 0 & \cdots & h_{L-1, N-L} & \cdots & h_{1, N-2} & h_{0, N-1}
\end{array}\right]}
\end{aligned}
$$

In this matrix, the top-right components are related to the channel response during the $\mathrm{CP}$ transmission timing. Thus, when considering column vectors in $\boldsymbol{H}$, the last $(L-1)$ vectors contains channel responses at different timings. To ease the following discussion, we employ an approximation in the top right part of $\boldsymbol{H}$ as

$$
\begin{aligned}
& H \simeq \\
& {\left[\begin{array}{ccccccc}
h_{0,0} & 0 & \cdots & 0 & \cdots & h_{2, N-2} & h_{1, N-1} \\
h_{1,0} & h_{0,1} & \cdots & 0 & \cdots & h_{3, N-2} & h_{2, N-1} \\
\vdots & \vdots & \ddots & \vdots & \ddots & \vdots & \vdots \\
h_{L-1,0} & h_{L-2,1} & \cdots & h_{0, N-L} & \cdots & 0 & 0 \\
\vdots & \vdots & \ddots & \vdots & \ddots & \vdots & \vdots \\
0 & 0 & \cdots & h_{L-2, N-L} & \cdots & h_{0, N-2} & 0 \\
0 & 0 & \cdots & h_{L-1, N-L} & \cdots & h_{1, N-2} & h_{0, N-1}
\end{array}\right]}
\end{aligned}
$$

Although the top-right components are replaced with different values, it is expected that the impact on the equivalent noise power is not severe when $L / N$ is reasonably small. Then, let us extract the cyclic matrix $\boldsymbol{H}_{\mathrm{c}}$ from $\boldsymbol{H}$. This matrix is given by

$$
\boldsymbol{H}_{\mathrm{c}}=\left[\begin{array}{ccccccc}
\bar{h}_{0} & 0 & \cdots & 0 & \cdots & \bar{h}_{2} & \bar{h}_{1} \\
\bar{h}_{1} & \bar{h}_{0} & \cdots & 0 & \cdots & \bar{h}_{3} & \bar{h}_{2} \\
\vdots & \vdots & \ddots & \vdots & \ddots & \vdots & \vdots \\
\bar{h}_{L-1} & \bar{h}_{L-2} & \cdots & \bar{h}_{0} & \cdots & 0 & 0 \\
\vdots & \vdots & \ddots & \vdots & \ddots & \vdots & \vdots \\
0 & 0 & \cdots & \bar{h}_{L-2} & \cdots & \bar{h}_{0} & 0 \\
0 & 0 & \cdots & \bar{h}_{L-1} & \cdots & \bar{h}_{1} & \bar{h}_{0}
\end{array}\right]
$$

where

$$
\bar{h}_{l}=\frac{1}{N} \sum_{i=0}^{N-1} h_{l, i} .
$$

With $\boldsymbol{H}_{\mathrm{c}}$ and the residual (non-cyclic) component $\boldsymbol{H}_{\mathrm{nc}}$ defined as $\boldsymbol{H}_{\mathrm{nc}}=\boldsymbol{H}-\boldsymbol{H}_{\mathrm{c}}$, the diagonal matrix $\boldsymbol{D}$ and the nondiagonal matrix $\boldsymbol{E}$ in (9) are expressed by

$$
\begin{gathered}
\boldsymbol{D}=\boldsymbol{F} \boldsymbol{H}_{\mathrm{c}} \boldsymbol{F}^{H} \\
\boldsymbol{E}=\boldsymbol{F} \boldsymbol{H}_{\mathrm{nc}} \boldsymbol{F}^{H} .
\end{gathered}
$$

Using expansion forms: $\boldsymbol{H}_{\mathrm{nc}}=\left[\boldsymbol{h}_{\mathrm{nc}, 0}, \ldots, \boldsymbol{h}_{\mathrm{nc}, N-1}\right]$ and $\boldsymbol{F}=$ $\left[\boldsymbol{f}_{0}, \ldots, \boldsymbol{f}_{N-1}\right]^{T}$, we can express $\boldsymbol{e}_{k}^{H} \boldsymbol{e}_{k}$ as

$$
\boldsymbol{e}_{k}^{H} \boldsymbol{e}_{k}=\left|\boldsymbol{f}_{k}^{T} \boldsymbol{h}_{\mathrm{nc}, 0}\right|^{2}+\left|\boldsymbol{f}_{k}^{T} \boldsymbol{h}_{\mathrm{nc}, 1}\right|^{2}+\cdots+\left|\boldsymbol{f}_{k}^{T} \boldsymbol{h}_{\mathrm{nc}, N-1}\right|^{2} .
$$

Define the vector time-shifted by $j$ symbols from $\boldsymbol{h}_{\mathrm{nc}, j}$ as $\boldsymbol{h}_{\mathrm{nc}, j}^{\prime}=\left[h_{\mathrm{nc}, 0, j}, \ldots, h_{\mathrm{nc}, L-1, j}, 0, \ldots, 0\right]^{T}$. Considering the time shifting property in Fourier transform, we have the following relation

$$
\left|\boldsymbol{f}_{k}^{T} \boldsymbol{h}_{\mathrm{nc}, j}\right|^{2}=\left|\boldsymbol{f}_{k}^{T} \boldsymbol{h}_{\mathrm{nc}, j}^{\prime}\right|^{2} .
$$

By defining the channel response vector at the $j$ th symbol timing as $\boldsymbol{h}_{j}=\left[h_{0, j}, \ldots, h_{L-1, j}, 0, \ldots, 0\right]^{T}$ and the channel response vector composed of the elements in $\boldsymbol{H}_{\mathrm{c}}$ as $\overline{\boldsymbol{h}}=$ $\left[\bar{h}_{0}, \ldots, \bar{h}_{L-1}, 0, \ldots, 0\right]^{T}, \boldsymbol{h}_{\mathrm{nc}, j}^{\prime}$ can be written as

$$
\boldsymbol{h}_{\mathrm{nc}, j}^{\prime}=\boldsymbol{h}_{j}-\overline{\boldsymbol{h}} \quad \text { for } j=0, \ldots, N-1 .
$$

If we can assume that a channel state changes linearly, $\boldsymbol{h}_{k}$ can be expressed by

$$
\boldsymbol{h}_{k}=\boldsymbol{h}_{0}+k \boldsymbol{\Delta},
$$


where $\Delta$ is a constant vector. Then, $\bar{h}$ can be rewritten as

$$
\overline{\boldsymbol{h}}=\frac{1}{N} \sum_{k=0}^{N-1} \boldsymbol{h}_{k}=\boldsymbol{h}_{0}+\alpha \boldsymbol{\Delta},
$$

where $\alpha=(N-1) / 2$. Substituting (33) into (31) yields

$$
\boldsymbol{h}_{\mathrm{nc}, j}^{\prime}=(j-\alpha) \boldsymbol{\Delta} \quad \text { for } j=0, \ldots, N-1 .
$$

Here, let us define a vector $\boldsymbol{h}_{\Delta}$ expressing a difference between $\boldsymbol{h}_{0}$ and $\boldsymbol{h}_{N-1}$ as

$$
\begin{aligned}
\boldsymbol{h}_{\Delta} & =\boldsymbol{h}_{N-1}-\boldsymbol{h}_{0} \\
& =\boldsymbol{h}_{\mathrm{nc}, N-1}^{\prime}-\boldsymbol{h}_{\mathrm{nc}, 0}^{\prime} \\
& =(N-1) \boldsymbol{\Delta} .
\end{aligned}
$$

Then, $\boldsymbol{h}_{\mathrm{nc}, j}^{\prime}$ can be rewritten as

$$
\boldsymbol{h}_{\mathrm{nc}, j}^{\prime}=\frac{j-\alpha}{N-1} \boldsymbol{h}_{\Delta} \quad \text { for } j=0, \ldots, N-1,
$$

and then substituting (38) into (29) yields

$$
\begin{aligned}
\boldsymbol{e}_{k}^{H} \boldsymbol{e}_{k} & =\left(\frac{-\alpha}{N-1}\right)^{2}\left|\boldsymbol{f}_{k}^{T} \boldsymbol{h}_{\Delta}\right|^{2}+\left(\frac{1-\alpha}{N-1}\right)^{2}\left|\boldsymbol{f}_{k}^{T} \boldsymbol{h}_{\Delta}\right|^{2}+\ldots \\
& +\left(\frac{N-1-\alpha}{N-1}\right)^{2}\left|\boldsymbol{f}_{k}^{T} \boldsymbol{h}_{\Delta}\right|^{2} \\
& =\sum_{i=0}^{N-1}\left(\frac{i-\alpha}{N-1}\right)^{2}\left|\boldsymbol{f}_{k}^{T} \boldsymbol{h}_{\Delta}\right|^{2} \\
& =\beta\left|\boldsymbol{f}_{k}^{T} \boldsymbol{h}_{\Delta}\right|^{2}
\end{aligned}
$$

where $\beta=\sum_{i=0}^{N-1}\left(\frac{i-\alpha}{N-1}\right)^{2}$. Consequently, we obtain the equivalent noise power by the following approximation

$$
\sigma_{e, k}^{2}=\sigma^{2}+P_{s} \beta\left|\boldsymbol{f}_{k}^{T} \boldsymbol{h}_{\Delta}\right|^{2}
$$

\section{REFERENCES}

[1] L. J. Cimini, Jr., "Analysis and simulation of a digital mobile channel using orthogonal frequency division multiplexing," IEEE Trans. Commun., vol. 33, no. 7, pp. 665-675, July 1985.

[2] K. G. Paterson and V. Tarokh, "On the existence and construction of good codes with low peak-to-average power ratios," IEEE Trans. Inform. Theory, vol. 46, no. 6, pp. 1974-1987, Sept. 2000.

[3] D. Falconer, S. L. Ariyavisitakul, A. Benyamin-Seeyar, and B. Eidson, "Frequency domain equalization for single-carrier broadband wireless systems," IEEE Commun. Mag., vol. 40, no. 4, pp. 58-66, April 2002.

[4] T. Hwang and Y. (G.) Li, "Iterative cyclic prefix reconstruction for coded single-carrier systems with frequency-domain equalization (SC-FDE)," Proc. VTC2003-Spring, vol. 3, pp. 1841-1845, April 2003.

[5] Stamoulis, S. N. Diggavi and N. Al-Dhahir, "Intercarrier Interference in MIMO OFDM," IEEE Trans. Signal Processing, vol. 50, no. 10, pp. 2415-2464, Oct. 2002.

[6] L. Deneire, B. Gyselinckx, and M. Engels, "Training sequence versus cyclic prefix - a new look on single carrier communication," IEEE Commun. Lett., vol. 5, no. 7, pp. 292-294, July 2001.

[7] H. Witschnig, T. Mayer, A. Springer, A. Koppler, L. Maurer, M. Huemer, and R. Weigel, "A different look on cyclic prefix for SC/FDE," Proc. PIMRC '02, vol. 2, pp. 824-828, Sept. 2002.

[8] J. Coon, M. Sandell, M. Beach, and J. McGeehan, "Channel and noise variance estimation and tracking algorithms for unique-word based single-carrier systems," IEEE Trans. Wireless Commun., vol. 5, no. 6, pp. 1488-1496, June 2006.

[9] Y. Ogawa, K. Nishio, T. Nishimura, and T. Ohgane, "A MIMO-OFDM system for high-speed transmission," Proc. VTC2003-Fall, vol. 2, pp. 1523-1527, Sept. 2003.

[10] W. H. Press, B. P. Flannery, S. A. Teukolsky, W. T. Vetterling, Numerical Recipes in C: The Art of Scientific Computing, second ed., Cambridge University Press, 1992.

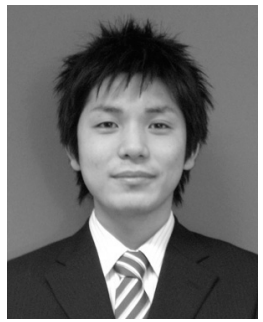

Keiichi Kambara received the B.E. degree from Hokkaido University, Sapporo, Japan, in 2006.

$\mathrm{He}$ is currently a graduate student toward the M.E. degree at the Graduate School of Information Science and Technology, Hokkaido University. His primary interests include high-mobility wireless systems and multiuser-MIMO systems.

Mr. Kambara received the IEEE VTS Japan Chapter Young Researcher's Encouragement Award in 2007.

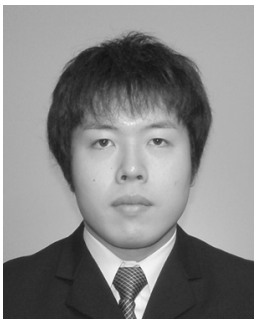

Hiroshi Nishimoto received the B.E., M.E., Ph.D. degrees from Hokkaido University, Sapporo, Japan, in 2003, 2005, and 2007, respectively.

$\mathrm{He}$ is currently a Post-Doctoral Fellow at the Graduate School of Information Science and Technology, Hokkaido University. His research interests are in MIMO propagation measurement and MIMO communication systems. He has been a Research Fellow of the Japan Society for the Promotion of Science since 2005.

Dr. Nishimoto received the IEEE VTS Japan Chapter Student Paper Award and the Young Researchers' Award of IEICE Japan, both in 2007.

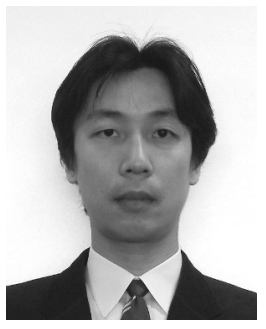

techniques.

Dr. Nishimura received the Young Researchers' Award of IEICE Japan in 2000 and the Best Paper Award from IEICE Japan in 2007.

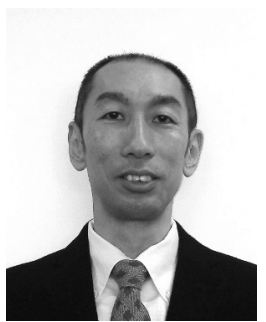

Takeo Ohgane received the B.E., M.E., and Ph.D. degrees in electronics engineering from Hokkaido University, Sapporo, Japan, in 1984, 1986, and 1994, respectively.

From 1986 to 1992, he was with Communications Research Laboratory, Ministry of Posts and Telecommunications. From 1992 to 1995, he was on assignment at ATR Optical and Radio Communications Research Laboratory. Since 1995, he has been with Hokkaido University, where he is an Associate Professor. During 2005-2006, he was at Centre for Communications Research, University of Bristol, U.K., as a Visiting Fellow. His interests are in MIMO signal processing for wireless communications.

Dr. Ohgane received the IEEE AP-S Tokyo Chapter Young Engineer Award in 1993, the Young Researchers' Award of IEICE Japan in 1990, and the Best Paper Award from IEICE Japan in 2007.

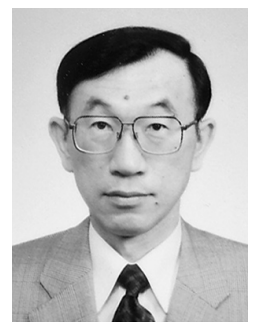

Yasutaka Ogawa received the B.E., M.E. and Ph.D. degrees from Hokkaido University, Sapporo, Japan, in 1973 , 1975, and 1978, respectively.

Since 1979, he has been with Hokkaido University, where he is currently a Professor of the Graduate School of Information Science and Technology. During 1992-1993, he was with ElectroScience Laboratory, the Ohio State University, U.S.A., as a Visiting Scholar, on leave from Hokkaido University. His interests are in adaptive antennas, mobile communications, super-resolution techniques, and

MIMO systems.

Dr. Ogawa received the Young Researchers' Award of IEICE Japan in 1982, and the Best Paper Award from IEICE Japan in 2007. 